\title{
Compound Fire Detection Algorithm Based on Fuzzy Neural Network
}

\author{
Yang Feng ${ }^{\mathrm{a}}$, Qu Na ${ }^{\mathrm{b}, *}$, Li Chao ${ }^{\mathrm{c}}$ \\ School of Safety Engineering, Shenyang Aerospace University, Shenyang 110136, China \\ a2495088176@qq.com, b11502332@qq.com, mn_qn@163.com
}

Keywords: fire detection; fuzzy neural network; MATLAB simulation; fire parameters

\begin{abstract}
This paper proposes a fuzzy neural network fire detection algorithm to improve the accuracy of fire detection. Taking the temperature, smoke concentration and CO concentration as the input of the system, the fuzzy neural network is used to analyze the multi input signals. Fuzzy neural network combines the advantages of neural network in finding the optimal solution at high speed and fuzzy system in dealing with fuzzy information and using existing knowledge. The effectiveness of the algorithm is proved by simulation with MATLAB toolbox.
\end{abstract}

\section{Introduction}

The fire detection technology is used to identify early fire through all kinds of fire phenomena (such as smoke, temperature, combustible gas). The tranditional and single fire detection technology is not accurate to detect the appearance of fire. It often occurs the problems of false negatives and false positives. Multi-parameter fire detection technology possesses powerful comprehensiveness and intelligence, it can also shorten the time of the detection and improve the accuracy. Multi-parameter fire detection technology has already become the focus in the field of research on firefighting. Document [1] used the multivariate analysis and the method of principal component analysis, ensured the amounts of element in the process of pattern identification, utilized three layers BP artificial neural network, formulated the multi-criteria discrimination rules by the $\mathrm{CO} 2$ and $\mathrm{CO}$ density and rise-rate. Document [2] utilized the online and off-line data in fire detection system, using fusion and complementation of the improved PCA (PCA), rough set (RS) theory and support vector machine (SVM). The fire information fusion is achieved from the data level, feature level and decision level. Document [3] designed a kind of wireless sensor network system based on Zigbee, which is applied to the intelligent home fire alarm system, in order to overcome the shortcomings of the traditional single fire detection system. Document [4] designed an intelligent distributed wireless fire detecting alarm system based on the control chip STM32. The system could collect the CO concentration, the concentrations of smoke and temperature simultaneously. It utilized the wireless communication technology to transmit data and adopted the intelligent algorithms to judge and deal with the fire information. Document[5] proposed two kinds of the indoor and outdoor fire probing method. The indoor detector was based on the depth and the amplitude image in the time-of-flight camera. The outdoor detector used the visual flame detector instead of rapidly varying depth detection. Document [6] proposed and evaluated two kinds of the computational method which was based on the methods about the mixture of information, when detecting fire in the rainforest.

In order to improve the accuracy of fire detection, the fuzzy neural network algorithm is used to fuse the fire parameters of temperature, smoke and CO.

\section{Fire Detection Algorithm}

In this paper, the structure of the fuzzy neural network applied to fire detection is shown in Fig.1.

The Input Layer. The process of material combustion is a kind of chemical reaction with smoke, heat and combustion gas. Combustion gases include carbon monoxide, carbon dioxide, etc. Different things in different environment produce different products, consequently in detection on the single fire parameter would occur the problems of omissive judgement and misinformation. In this paper, we compromise the fire parameters(such as smoke, temperature and the carbon monoxide) to make 
the alarm accurate. As shown in Figure 1, the first layer is the input layer, there are three-node signal: the smoke concentration, temperature and the CO concentration respectively.

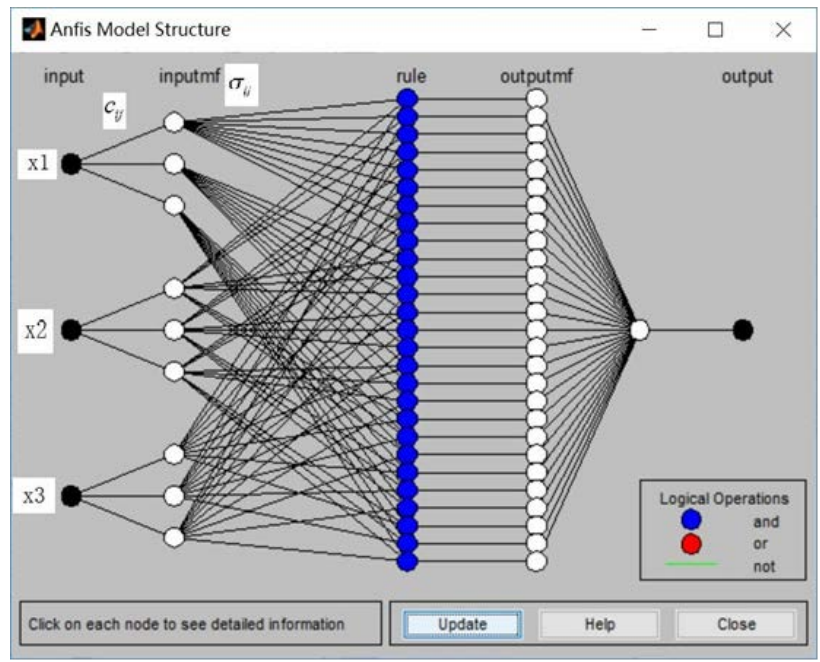

Fig.1 Schematic diagram of fuzzy neural network

The Fuzzy Layer. The second layer is the fuzzy layer, that the input quantity is fuzzed. According to the membership function, make raw input transformed by the grade of membership from 0 to 1 . For each input variable use the three quantity of language, they are small, medium and large respectively. Adopt the Gauss-Type Function, grade of membership is shown in formula 1:

$$
\mu=\exp \left[-\frac{1}{2}\left(\left(x-c_{i j}\right) / \sigma_{i j}\right)^{2}\right]
$$

In the formula, $i=1,2,3, j=1,2,3$. They are the center and width of the membership function, the node output from zero to one, which is the grade of membership in the quantity of language. The membership function is shown in the Fig. 2.

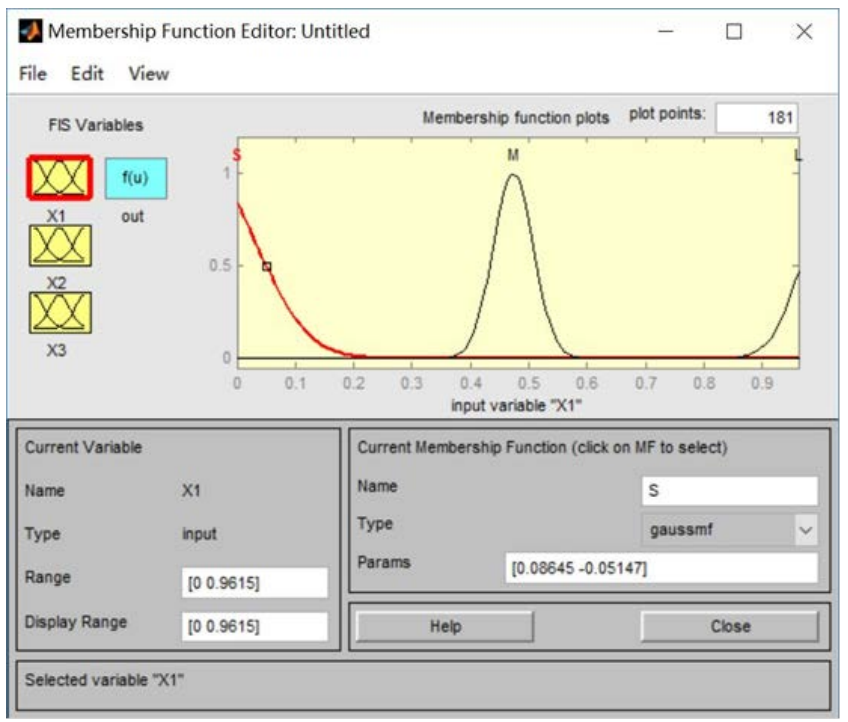

Fig.2 membership function graph

The Regularity Layer. The third layer is the accomplishment layer of fuzzy logic regularity matching. In this layer, each node represents a fuzzy regularity, which should contain all combination of input variables. The number of fuzzy regularity $(\mathrm{m})$ is the total number of nodes in this layer.

The signal of smoke X1, CO signal X2, and signal of temperature X3 s is used as input variable. The $m_{i}$ equals 3 and $m$ is 27 of the number of fuzzy regularities. Part of rules is shown in the Fig.3.

The Normalized Layer. The forth is normalized layer.

$$
y_{k}=\frac{\mu_{k}}{\sum_{p=1}^{m} \mu_{p}}, k=1,2
$$


In this layer, the total number of node is the same of that in the third. It accomplishes that normalizes the output of the third layer, which is the preparation for solution of the third layer.

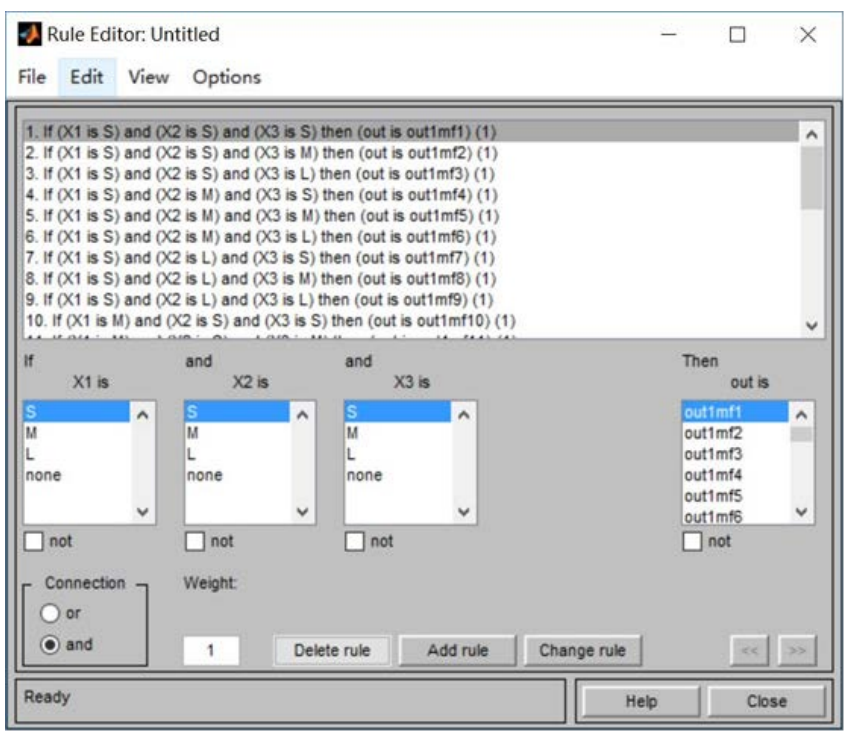

Fig.3 Schematic diagram of regularity

The Output Layer. The fifth layer is the output layer.

$$
y_{t}=\sum_{k} \omega_{k t} y_{k}
$$

The error function is shown by formula (4).

$$
E=\frac{\sum_{t=1}^{n}\left(\overline{y_{t}}-y_{t}\right)^{2}}{2}
$$

In which the actual output of the neural network and the expected output, E represents the error function of a set of learning mode.

\section{MATLAB Simulation}

The software MATLAB provides a toolbox of the neural network. This fire detection model has three layers BP network and three input nodes which are temperature, smoke concentration and gas concentration. There are twenty seven nodes in the middle layer and one node in the output layer. The experimental data of fire is used as the training samples of neural network. The output of the model is the expected probability of fire. In Fig. 4, we can see that network meets the conditions set after 3000 training.

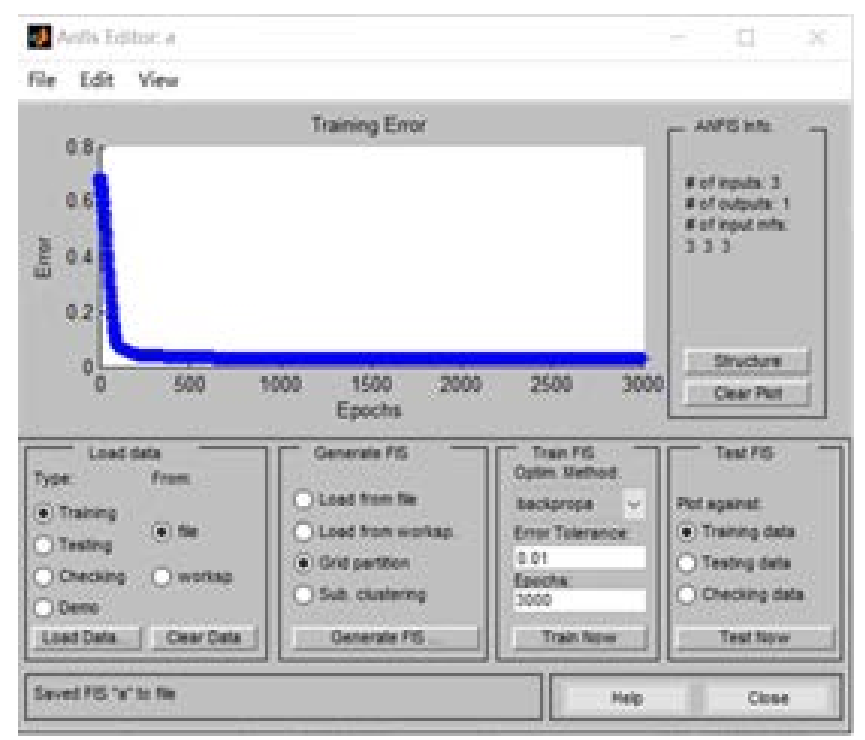

Fig. 4 Neural network training 
The comparison calculated by the neural network between expected value and the value of actual output is shown in the Table 1. The fuzzy rule is shown in Fig.5.

Table 1 Sample data and expected output

\begin{tabular}{ccccccc}
\hline $\begin{array}{c}\text { group } \\
\text { number }\end{array}$ & temperature & smoke & CO & $\begin{array}{c}\text { fire } \\
\text { probability }\end{array}$ & $\begin{array}{c}\text { expected } \\
\text { probability }\end{array}$ & error \\
\hline 1 & 0.9615 & 0.0755 & 0.7391 & 0.809 & 0.8128 & 0.0038 \\
2 & 0.9231 & 0.1321 & 0.7826 & 0.824 & 0.8265 & 0.0025 \\
3 & 0.8462 & 0.0755 & 0.7826 & 0.811 & 0.8128 & 0.0017 \\
4 & 0.8846 & 0.1321 & 0.8261 & 0.835 & 0.8265 & 0.0085 \\
5 & 0.8462 & 0.1132 & 0.913 & 0.829 & 0.8210 & 0.008 \\
6 & 0.8462 & 0.0943 & 1 & 0.821 & 0.8164 & 0.0046 \\
7 & 0.8462 & 0.0943 & 0.7826 & 0.816 & 0.8164 & 0.0004 \\
8 & 0.8615 & 0.0943 & 0.6957 & 0.813 & 0.8164 & 0.0034 \\
9 & 0.8077 & 0.0943 & 0.4348 & 0.812 & 0.8164 & 0.0044 \\
\hline
\end{tabular}

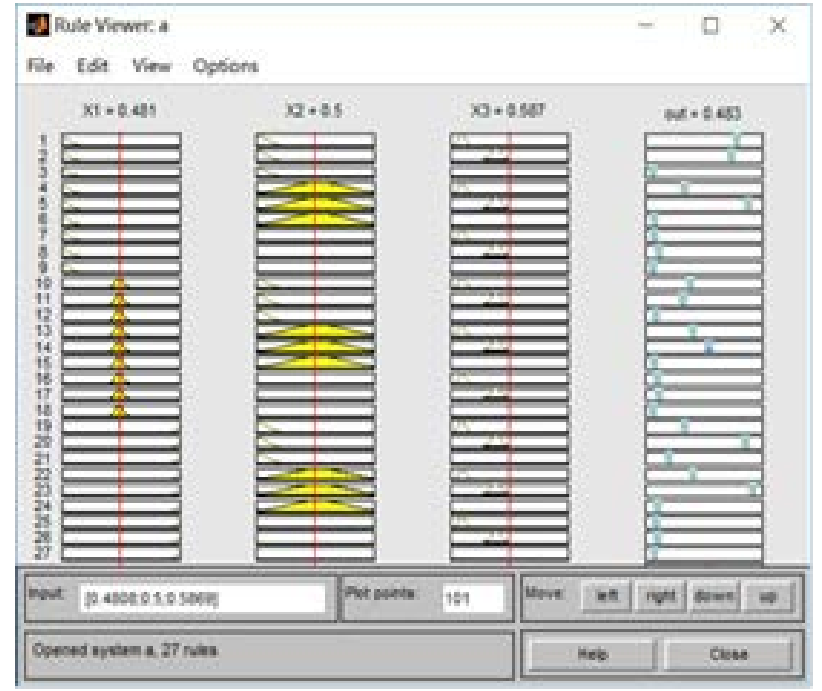

Fig. 5 Fuzzy rule

\section{Summary}

In order to detect the fire more accurately, the content about this essay is researching on algorithm of the fire detection based on fuzzy neural network. We had come to following conclusions.

(1)In basis of fire alarm combined with typical fire and the process of the multi-data fusion technology, this paper proposes the data fusion technology of intelligent algorithm. This algorithm combines neural network and special fuzzy logic, which can determine the fire more accurately and ensure some interference signals.

(2)The fuzzy logic possesses an ability of strong comprehensive judgment and it is suitable for fire detection and alarm system. The functions and commands in toolbox of fuzzy logic accomplish the algorithm of fire detecting system, then design the fuzzy controller.

(3) This paper establishes a neural network model for fire detection through neural network and completes the network training and system simulation.

\section{Acknowledgement}

In this paper, the research was sponsored by College Students Training Program of Innovation and Entrepreneurship in Shenyang Aerospace University (DS504101).

\section{References}

[1]Tang Zhenghua, Wang Shu, Chen Tao. Application of multisensor/multicriteria detector in fire detection. Journal of Transducer Technology, 2001, 20(3):33-35 
[2] Zhang Xin, Sui Jinxue, Zhang Yan. Study on the application of information fusion technology in fire detection. China Safety Science Journal, 2011, 21(6):95-98

[3] Xu Zhi, Li Xinchun, Zhang Lei. Multi-sensor fire early-warning system based on ZigBee. Journal of Xihua University (Natural Science), 2012, (31)6:73-76

[4] Hu Zhuge, Zhao Minhua. A wireless fire detection and alarm system based on the information fusion technology. Electronic Sci. \& Tech., 2012, (25)10:36-39

[5] Steven Verstockt, Sofie Van Hoecke. Multi-modal time-of-flight based fire detection. Multimed Tools Appl, 2014, 69:313-338

[6] Da Penha Jr., Osman, Nakamura, Eduardo. Fusing light and temperature data for fire detection. 15th IEEE Symposium on Computers and Communications, 2010: 107-112 